\title{
On type-2 soft topologies
}

\author{
Sk. Nazmul \\ Department of Mathematics, Bankura University, Purandarpur, Bankura-722155, West Bengal, India
}

Received: 6 October 2016, Accepted: 1 February 2017

Published online: 13 November 2017.

\begin{abstract}
In the present paper, a notion of type-2 soft topology is introduced and some of its important properties are studied. For this we have defined product of type- 2 soft sets, type- 2 soft product spaces, type- 2 soft continuous mappings etc. and their topological behaviours are examined.
\end{abstract}

Keywords: Soft sets, soft mappings, soft topologies, type-2 soft sets, type-2 soft mappings, type-2 soft topologies.

\section{Introduction}

In natural occurance of events, uncertainty is the most prevalent aspect and several theories were developed to deal with it. Probability theory deals with the uncertainties caused by the randomness of a situation but it can not handle other type of situations where the uncertainties evolving out of imprecise informations, limitations of computability and perceptibility etc. L. A. Zadeh [18], in 1965 introduced fuzzy set theory (FST) and attempted to formalize this grey area. Afterwards many generalizations, modifications and extensions of FST came up with different perspectives and several research works were published on fuzzy subgroups, fuzzy topological spaces, fuzzy topological groups etc. In 1975, Zadeh [19] coined the notion of type-2 fuzzy sets which are characterized by fuzzy membership functions that are themselves type-1 fuzzy in nature. In the theory of fuzzy set as well as type-2 fuzzy sets, membership function play the main role and this function depends on various factors and because of this many complexities are encountered while dealing with it. In 1999, D. Molodtsov [11] proposed soft set as an alternative way. He took parameterized family of sets instead of membership function. He also proved that Zadeh's fuzzy sets were spacial types of soft sets. Therefore, it might be stated that in the field of applications in real life problems the parameterization approach of soft set theory is better user friendly as compared to the membership function approach of fuzzy set theory. Since then research activities are going on with all these theories in their pure form as well as in their hybridizations. Currently research on soft set theory are progressing at a very fast pace and in areas involving soft groups [1], soft mappings [7,9], soft topology [3,5, $6,17]$, soft topological groups $[13,14]$ etc. which are applied to decision making problems including texture classification, data analysis etc.

Recently in 2015, Rajashi Chatterjee et al. [4] proposed a parameterized structure for type-2 fuzzy sets and named it type-2 soft sets. As a continuation and observing a huge potential of soft set theory, it is natural to investigate the behaviour of the topological structures in type- 2 soft set settings. In this paper, we have introduced a notion of type-2 soft topologies and investigate some of its important properties. 


\section{Preliminaries}

In this section, following $[7,8,11,15,16]$, some definitions and results of soft sets, soft mappings and type-2 soft mappings are given. Unless otherwise stated, $X$ will be assumed to be an initial universal set, $E$ will be taken to be a set of parameters, $P(X)$ denote the power set of $X$.

Definition 1. [11,8] A pair $(F, A)$ where $F$ is a mapping from $A \subseteq E$ to $P(X)$, is called a soft set or type -1 soft set over $X$. Let $S_{1}(X, E)$ denotes the set of all soft sets or type-1 soft sets over $X$ under the parameter $E$.

Definition 2. Let $(F, A),(G, B) \in S_{1}(X, E)$. Then

(i) $(F, A)$ is said to be soft subset of $(G, B)$ if $A \subseteq B$ and $F(\alpha) \subseteq G(\alpha), \forall \alpha \in A$. This relation is denoted by $(F, A) \simeq(G, B)[11,8]$.

(ii) The complement of a soft set $(F, A)$ is defined as $(F, A)^{c}=\left(F^{c}, A\right)$, where $F^{c}(\alpha)=(F(\alpha))^{c}=X-F(\alpha), \forall \alpha \in A$ $[11,8]$.

(iii) $(F, A)$ is said to be a null soft set (an absolute soft set) if $F(\alpha)=\phi(F(\alpha)=X), \forall \alpha \in A$. This is denoted by $(\widetilde{\phi}, A)((\widetilde{X}, A))[10]$.

Definition 3. [11,8] Let $(F, A),(G, B) \in S_{1}(X, E)$. Then their

(a) Union, is a soft set $(H, A \cup B) \in S_{1}(X, E)$, denoted by $(F, A) \widetilde{\cup}(G, B)=(H, A \cup B)$, is defined by $\forall \alpha \in(A \cup B)$

$$
H(\alpha)= \begin{cases}F(\alpha) & \text { if } \alpha \in(A-B) \\ G(\alpha) & \text { if } \alpha \in(B-A) \\ F(\alpha) \cup G(\alpha) & \text { if } \alpha \in(A \cap B)\end{cases}
$$

(b) Intersection, is a soft set $(H, A \cap B) \in S_{1}(X, E)$, denoted by $(F, A) \widetilde{\cap}(G, B)=(H, A \cap B)$, is defined by $H(\alpha)=$ $F(\alpha) \cap G(\alpha), \forall \alpha \in(A \cap B)$.

(c) AND, is a soft set $(H, A \times B) \in S(X, E \times E)$, denoted by $(F, A) \widetilde{\wedge}(G, B)=(H, A \times B)$, is defined by $H(\alpha, \beta)=$ $F(\alpha) \cap G(\beta), \forall(\alpha, \beta) \in(A \times B)$.

(d) OR, is a soft set $(H, A \times B) \in S(X, E \times E)$, denoted by $(F, A) \widetilde{V}(G, B)=(H, A \times B)$, is defined by $H(\alpha, \beta)=F(\alpha) \cup$ $G(\beta), \forall(\alpha, \beta) \in(A \times B)$.

Remark. [10] For any $(F, A) \in S_{1}(X, E)$, construct a soft set $(H, E) \in S_{1}(X, E)$, where $\forall \alpha \in E$,

$$
H(\alpha)= \begin{cases}F(\alpha) & \text { if } \alpha \in A \\ \phi & \text { if } \alpha \in E / A\end{cases}
$$

Thus the soft sets $(F, A)$ and $(H, E)$ are equivalent to each other and the usual set operations of the soft sets $\left(F_{i}, A_{i}\right) \in$ $S_{1}(X, E), i \in \Delta$ is the same as the corresponding soft sets $\left(H_{i}, E\right) \in S_{1}(X, E), i \in \Delta$.

So, in this paper we have considered the soft sets over the same parameter set $A$.

Definition 4. [7] Let $S_{1}(X, A)$ and $S_{1}(Y, B)$ be the families of all soft sets over $X$ and $Y$ respectively. The mapping $f_{\varphi}: S_{1}(X, A) \rightarrow S_{1}(Y, B)$ is called a soft mapping from $X$ to $Y$, where $f: X \rightarrow Y$ and $\varphi: A \rightarrow B$ are two mappings. Also

(i) the image of a soft set $(F, A) \in S_{1}(X, A)$ under the mapping $f_{\varphi}$ is denoted by $f_{\varphi}[(F, A)]=\left(f_{\varphi}(F), B\right)$, and is defined by $\forall \beta \in B$,

$$
\left[f_{\varphi}(F)\right](\beta)= \begin{cases}\bigcup_{\alpha \in \varphi^{-1}(\beta)}[f[F(\alpha)]] & \text { if } \varphi^{-1}(\beta) \neq \phi \\ \phi & \text { otherwise }\end{cases}
$$

(ii) the inverse image of a soft set $(G, B) \in S_{1}(Y, B)$ under the mapping $f_{\varphi}$ is denoted by $f_{\varphi}^{-1}[(G, B)]=\left(f_{\varphi}^{-1}(G), A\right)$, and is defined by $\left[f_{\varphi}^{-1}(G)\right](\alpha)=f^{-1}[G[\varphi(\alpha)]], \forall \alpha \in A$. 
(iii) The soft mapping $f_{\varphi}$ is called injective (surjective) if $f$ and $\varphi$ are both injcetive (surjective).

(iv) The soft mapping $f_{\varphi}$ is identity soft mapping, if $f$ and $\varphi$ are both clasical identity mappings.

Proposition 1. [7] Let $X$ and $Y$ be two nonempty sets and $f_{\varphi}: S_{1}(X, A) \rightarrow S_{1}(Y, B)$ be a soft mapping. If $(F, A),\left(F_{i}, A\right) \in$ $S_{1}(X, A)$ and $(G, B),\left(G_{i}, B\right) \in S_{1}(Y, B), i \in \Delta$ then

(i) $(F, A) \widetilde{\subseteq} f_{\varphi}^{-1}\left[f_{\varphi}(F, A)\right]$, the equality holds if $f_{\varphi}$ is injective.

(ii) $f_{\varphi}\left[f_{\varphi}^{-1}(G, B)\right] \widetilde{\subseteq}(G, B)$, the equality holds if $f_{\varphi}$ is surjective.

(iii) $] f_{\varphi}\left[\widetilde{\bigcup}_{i \in \Delta}\left(F_{i}, A\right)\right]=\widetilde{\bigcup}_{i \in \Delta}\left[f_{\varphi}\left(F_{i}, A\right)\right]$, and $f_{\varphi}^{-1}\left[\widetilde{\bigcup}_{i \in \Delta}\left(G_{i}, A\right)\right]=\widetilde{\bigcup}_{i \in \Delta}\left[f_{\varphi}^{-1}\left(G_{i}, A\right)\right]$;

(iv) $f_{\varphi}\left[\widetilde{\bigcap}_{i \in \Delta}\left(F_{i}, A\right)\right] \widetilde{\subseteq} \widetilde{\bigcap}_{i \in \Delta}\left[f_{\varphi}\left(F_{i}, A\right)\right]$, the equality holds if $f_{\varphi}$ is injective and $f_{\varphi}^{-1}\left[\widetilde{\bigcap}_{i \in \Delta}\left(G_{i}, A\right)\right]=\widetilde{\bigcap}_{i \in \Delta}\left[f_{\varphi}^{-1}\left(G_{i}, A\right)\right]$.

Definition 5. [14] A soft set $(F, A)$ in $S_{1}(X, A)$ is said to be a

(i) constant soft set if $F(\alpha)=X_{0} \subseteq X, \forall \alpha \in A$. It is denoted by $\left(\widetilde{X_{0}}, A\right)$.

(ii) pseudo constant soft set if $F(\alpha)=X$ or $\phi, \forall \alpha \in A$. The set of all pseudo constant soft set over $X$ is denoted by $C S_{1}(X, A)$.

Proposition 2. [14] Let $X$ and $Y$ be two nonempty sets and $f_{\varphi}: S_{1}(X, A) \rightarrow S_{1}(Y, B)$ be a soft mapping. If $(F, A) \in S_{1}(X, A)$ and $(G, B) \in S_{1}(Y, B)$ then

(i) $(G, B) \in C S_{1}(Y, B) \Rightarrow f_{\varphi}^{-1}[(G, B)] \in C S_{1}(X, A)$;

(ii) $(F, A) \in C S_{1}(X, A)$ and $f$ is surjective $\Rightarrow f_{\varphi}[(F, A)] \in C S_{1}(Y, B)$.

Definition 6. [15] A soft set $(E, A) \neq(\widetilde{\phi}, A)$ over $X$ is called a

(i) generalized soft element (or briefly g-soft element) of $X$, if $E(\alpha)=\phi$ or $\exists x \in X$ such that $E(\alpha)=\{x\}, \forall \alpha \in A$.

(ii) constant $g$ - soft element of $X$, if $\exists x \in X$ such that $E(\alpha)=\{x\}, \forall \alpha \in A$.

(iii) pseudo constant $g-$ soft element of $X$, if $\exists x \in X$ such that $E(\alpha)=\phi$ or $\{x\}, \forall \alpha \in A$.

We denotes $E_{x}$, the constant soft element defined by $E(\alpha)=\{x\}, \forall \alpha \in A$ and $E_{x}^{\alpha}$, the soft element defined by $E(\alpha)=\{x\}$ and $E(\beta)=\phi, \forall \beta(\neq \alpha) \in A$.

Definition 7. [15] The soft mapping $f_{\varphi}: S_{1}(X, A) \rightarrow S_{1}(Y, B)$ is called constant if $\exists$ a soft element $(E, B) \in S_{1}(Y, B)$ such that $f_{\varphi}[(F, A)]=(E, B), \forall(F, A)[\neq(\widetilde{\phi}, A)] \in S_{1}(X, A)$.

Proposition 3. [15] The soft mapping $f_{\varphi}: S_{1}(X, A) \rightarrow S_{1}(Y, B)$ is a constant iff the mappings $f: X \rightarrow Y$ and $\varphi: A \rightarrow B$ are constant.

Proposition 4. [15] Let $f_{\varphi}: S_{1}(X, A) \rightarrow S_{1}(Y, B)$ be a soft mapping. If the mapping $\varphi: A \rightarrow B$ be constant then, $f_{\varphi}^{-1}[(G, B)]$ is a constant soft set $\forall(G, B) \in S_{1}(Y, B)$.

Definition 8. [2] Let $(F, A) \in S_{1}(X, A)$ and $(G, B) \in S_{1}(Y, B)$. The Cartesian product of $(F, A)$ and $(G, B)$ is denoted by $(F, A) \widetilde{\times}(G, B)=(F \widetilde{\times} G, A \times B) \in S(X \times Y, A \times B)$ and defined as $\forall(\alpha, \beta) \in A \times B,(F \widetilde{\times} G)(\alpha, \beta)=F(\alpha) \times G(\beta)$.

Proposition 5. Let $(F, A),\left(F_{1}, A\right),\left(F_{2}, A\right) \in S_{1}(X, A)$ and $(G, B),\left(G_{1}, B\right),\left(G_{2}, B\right),(H, B) \in S_{1}(Y, B)$. Then

(i) $(F, A) \widetilde{\times}[(G, B) \widetilde{\cup}(H, B)]=[(F, A) \widetilde{\times}(G, B)] \widetilde{\cup}[(F, A) \widetilde{\times}(H, B)]$;

(ii) $(F, A) \widetilde{\times}[(G, B) \widetilde{\cap}(H, B)]=[(F, A) \widetilde{\times}(G, B)] \widetilde{\cap}[(F, A) \widetilde{\times}(H, B)]$;

(iii) $]\left[\left(F_{1}, A\right) \widetilde{\times}\left(G_{1}, B\right)\right] \widetilde{\cap}\left[\left(F_{2}, A\right) \widetilde{\times}\left(G_{2}, B\right)\right]=\left[\left(F_{1}, A\right) \widetilde{\cap}\left(F_{2}, A\right)\right] \widetilde{\times}\left[\left(G_{1}, B\right) \widetilde{\cap}\left(G_{2}, B\right)\right]$.

Proof. (i) Since,

$$
(F, A) \widetilde{\times}[(G, B) \widetilde{\cup}(H, B)]=(F, A) \widetilde{\times}[G \widetilde{\cup} H, B]=[F \widetilde{\times}(G \widetilde{\cup} H), A \times B] .
$$


So, for each $(\alpha, \beta) \in A \times B$,

$$
\begin{aligned}
{[F \widetilde{\times}(G \widetilde{\cup} H)](\alpha, \beta) } & =F(\alpha) \times[G(\beta) \cup H(\beta)] \\
& =[F(\alpha) \times G(\beta)] \cup[F(\alpha) \times H(\beta)] \\
& =[(F, A) \widetilde{\times}(G, B)](\alpha, \beta) \cup[(F, A) \widetilde{\times}(H, B)](\alpha, \beta) \\
& =[[(F, A) \widetilde{\times}(G, B)] \widetilde{\cup}[(F, A) \widetilde{\times}(H, B)]](\alpha, \beta) .
\end{aligned}
$$

Thus $[F \tilde{\times}(G \widetilde{\cup} H), A \times B]=[(F, A) \widetilde{\times}(G, B)] \widetilde{\cup}[(F, A) \tilde{\times}(H, B)]$

Therefore $(F, A) \widetilde{\times}[(G, B) \widetilde{U}(H, B)]=[(F, A) \widetilde{\times}(G, B)] \widetilde{\cup}[(F, A) \widetilde{\times}(H, B)]$.

(ii) Proof is similar to part (i).

(iii) For any $(\alpha, \beta) \in A \times B$, we have

$$
\begin{aligned}
{\left[\left[\left(F_{1}, A\right) \widetilde{\times}\left(G_{1}, B\right)\right] \widetilde{\cap}\right.} & {\left.\left[\left(F_{2}, A\right) \widetilde{\times}\left(G_{2}, B\right)\right]\right](\alpha, \beta) } \\
& =\left[\left(F_{1}, A\right) \widetilde{\times}\left(G_{1}, B\right)\right](\alpha, \beta) \cap\left[\left(F_{2}, A\right) \widetilde{\times}\left(G_{2}, B\right)\right](\alpha, \beta) \\
& =\left[F_{1}(\alpha) \times G_{1}(\beta)\right] \cap\left[F_{2}(\alpha) \times G_{2}(\beta)\right] \\
& =\left[F_{1}(\alpha) \cap F_{2}(\alpha)\right] \times\left[G_{1}(\beta) \cap G_{2}(\beta)\right] \\
& =\left[F_{1} \widetilde{\cap} F_{2}\right](\alpha) \times\left[G_{1} \widetilde{\cap} G_{2}\right](\beta) \\
& =\left[\left(F_{1} \widetilde{\cap} F_{2}, A\right) \widetilde{\times}\left(G_{1} \widetilde{\cap} G_{2}, B\right)\right](\alpha, \beta) \\
& =\left[\left[\left(F_{1}, A\right) \widetilde{\cap}\left(F_{2}, A\right)\right] \widetilde{\times}\left[\left(G_{1}, B\right) \widetilde{\cap}\left(G_{2}, B\right)\right]\right](\alpha, \beta)
\end{aligned}
$$

Therefore $\left[\left(F_{1}, A\right) \widetilde{\times}\left(G_{1}, B\right)\right] \widetilde{\cap}\left[\left(F_{2}, A\right) \widetilde{\times}\left(G_{2}, B\right)\right]=\left[\left(F_{1}, A\right) \widetilde{\cap}\left(F_{2}, A\right)\right] \widetilde{\times}\left[\left(G_{1}, B\right) \widetilde{\cap}\left(G_{2}, B\right)\right]$.

Type-2 soft sets: Following [4], the definition and operations of Type-2 soft sets are given first and then we have introduced type-2 soft mappings and study some of its properties.

Definition 9. [4] The pair $[\mathscr{F}, A]$ where $\mathscr{F}$ is a mapping from $A$ to $S_{1}(X, A)$ is called type-2 soft set over $(X, A)$. In this case, corresponding to each parameter $\alpha \in A, \exists$ a type-1 soft set $\left(F_{\alpha}, A\right)$ (say) over $(X, A)$ such that $\mathscr{F}(\alpha)=\left(F_{\alpha}, A\right)$ where $F_{\alpha}: A \rightarrow P(X)$. Also, $S_{2}(X, A)$ denotes the set of all type-2 soft sets over $(X, A)$.

Definition 10. [4] Let $[\mathscr{F}, A],[\mathscr{G}, A] \in S_{2}(X, A)$. Then

(i) $[\mathscr{F}, A]$ is said to be soft subset of $[\mathscr{G}, A]$ if $\mathscr{F}(\alpha) \widetilde{\subseteq} \mathscr{G}(\alpha), \forall \alpha \in A$. This relation is denoted by $[\mathscr{F}, A] \sqsubseteq[\mathscr{G}, A]$.

(ii) the complement of a type-2 soft set $[\mathscr{F}, A]$ is defined as $[\mathscr{F}, A]^{c}=\left[\mathscr{F}^{c}, A\right]$, where $\mathscr{F}^{c}(\alpha)=[\mathscr{F}(\alpha)]^{c}, \forall \alpha \in A$.

(iii) $[\mathscr{F}, A]$ is said to be a type-2 null soft set (absolute soft set) if $\mathscr{F}(\alpha)=(\widetilde{\phi}, A)(\mathscr{F}(\alpha)=(\widetilde{X}, A)), \forall \alpha \in A$. This is denoted by $[\widetilde{\Phi}, A]([\widetilde{\mathscr{X}}, A])$.

Definition 11. [4] Let [ $\mathscr{F}, A],[\mathscr{G}, A] \in S_{2}(X, A)$. Then their

(a) Union (Intersection), is a type-2 soft set $[\mathscr{H}, A]$, denoted by $[\mathscr{F}, A] \sqcup[\mathscr{G}, A]=[\mathscr{H}, A]([\mathscr{F}, A] \sqcap[\mathscr{G}, A]=[\mathscr{H}, A])$, is defined by $\mathscr{H}(\alpha)=\mathscr{F}(\alpha) \widetilde{\cup} \mathscr{G}(\alpha)(\mathscr{H}(\alpha)=\mathscr{F}(\alpha) \widetilde{\cap} \mathscr{G}(\alpha)), \forall \alpha \in A$.

(b) AND $($ OR), is a type-2 soft set $[\mathscr{H}, A \times A]$, denoted by $[\mathscr{F}, A] \wedge[\mathscr{G}, A]=[\mathscr{H}, A \times A]([\mathscr{F}, A] \vee[\mathscr{G}, A]=[\mathscr{H}, A \times A])$, is defined by $\mathscr{H}(\alpha, \beta)=\mathscr{F}(\alpha) \widetilde{\cap} \mathscr{G}(\beta)(\mathscr{H}(\alpha, \beta)=\mathscr{F}(\alpha) \widetilde{\cup} \mathscr{G}(\beta)), \forall(\alpha, \beta) \in(A \times A)$.

Definition 12. [16] Let $S_{2}(X, A)$ and $S_{2}(Y, B)$ be the families of all type-2 soft sets over $(X, A)$ and $(Y, B)$ respectively. The mapping $f_{\varphi_{\psi}}: S_{2}(X, A) \rightarrow S_{2}(Y, B)$ is called a type-2 soft mapping, where $f_{\varphi}: S_{1}(X, A) \rightarrow S_{1}(Y, B)$ is a type-1 soft mapping and $\psi: A \rightarrow B$ is a mapping. Also 
(i) the image of a type-2 soft set $[\mathscr{F}, A] \in S_{2}(X, A)$ under the mapping $f_{\varphi_{\psi}}$ is denoted by $f_{\varphi_{\psi}}[[\mathscr{F}, A]]=\left[f_{\varphi_{\psi}}(\mathscr{F}), B\right]$, and is defined by $\forall \beta \in B$,

$$
\left[f_{\varphi_{\psi}}(\mathscr{F})\right](\beta)= \begin{cases}\widetilde{U}_{\alpha \in \psi^{-1}(\beta)}\left[f_{\varphi}[\mathscr{F}(\alpha)]\right] & \text { if } \psi^{-1}(\beta) \neq \phi \\ (\widetilde{\phi}, B) & \text { otherwise }\end{cases}
$$

(ii) the inverse image of a type-2 soft set $[\mathscr{G}, B) \in S_{2}(Y, B)$ under the mapping $f_{\varphi_{\psi}}$ is denoted by $f_{\varphi}^{-1}[[G, B]]=\left[f_{\varphi}^{-1}(\mathscr{G}), A\right]$, and is defined by $\left[f_{\varphi}^{-1}(\mathscr{G})\right](\alpha)=f_{\varphi}^{-1}[\mathscr{G}[\psi(\alpha)]], \forall \alpha \in A$.

(iii) The soft mapping $f_{\varphi_{\psi}}$ is called injective (surjective) if $f_{\varphi}$ and $\psi$ are both injcetive (surjective).

(iv) The soft mapping $f_{\varphi_{\psi}}$ is identity soft mapping, if $f_{\varphi}$ and $\psi$ are both identity mappings.

Proposition 6. [16] Let $X$ and $Y$ be two nonempty sets and $f_{\varphi_{\psi}}: S_{2}(X, A) \rightarrow S_{2}(Y, B)$ be a type-2 soft mapping. If $[\mathscr{F}, A],\left[\mathscr{F}_{i}, A\right] \in S_{2}(X, A)$ and $[\mathscr{G}, B],\left[\mathscr{G}_{i}, B\right] \in S_{2}(Y, B), i \in \Delta$. Then

(i) $\left[\mathscr{F}_{1}, A\right] \sqsubseteq\left[\mathscr{F}_{2}, A\right] \Rightarrow f_{\varphi_{\psi}}\left(\left[\mathscr{F}_{1}, A\right]\right) \sqsubseteq f_{\varphi_{\psi}}\left(\left[\mathscr{F}_{2}, A\right]\right)$;

(ii) $\left[\mathscr{G}_{1}, B\right] \sqsubseteq\left[\mathscr{G}_{2}, B\right] \Rightarrow f_{\varphi_{\psi}}^{-1}\left(\left[\mathscr{G}_{1}, B\right]\right) \sqsubseteq f_{\varphi_{\psi}}^{-1}\left(\left[\mathscr{G}_{2}, B\right]\right)$;

(iii) $[\mathscr{F}, A] \sqsubseteq f_{\varphi_{\psi}}^{-1}\left(f_{\varphi_{\psi}}([\mathscr{F}, A])\right)$, the equality holds if $f_{\varphi_{\psi}}$ is injective.

(iv) $f_{\varphi_{\psi}}\left(f_{\varphi_{\psi}}^{-1}([\mathscr{G}, B])\right) \sqsubseteq[\mathscr{G}, B]$, the equality holds if $f_{\varphi_{\psi}}$ is surjective.

(v) $f_{\varphi_{\psi}}\left[\bigsqcup_{i \in \Delta}\left[\mathscr{F}_{i}, A\right]\right]=\bigsqcup_{i \in \Delta}\left[f_{\varphi_{\psi}}\left(\left[\mathscr{F}_{i}, A\right]\right)\right]$;

(vi) $f_{\varphi_{\psi}}\left[\sqcap_{i \in \Delta}\left[\mathscr{F}_{i}, A\right]\right] \sqsubseteq \Pi_{i \in \Delta}\left[f_{\varphi_{\psi}}\left(\left[\mathscr{F}_{i}, A\right]\right)\right]$, the equality holds if $f_{\varphi_{\psi}}$ is injective.

(vii) $f_{\varphi_{\psi}}^{-1}\left[\bigsqcup_{i \in \Delta}\left[\mathscr{G}_{i}, B\right]\right]=\bigsqcup_{i \in \Delta}\left[f_{\varphi_{\psi}}^{-1}\left(\left[\mathscr{G}_{i}, B\right]\right)\right]$;

(viii) $f_{\varphi_{\psi}}^{-1}\left[\cap_{i \in \Delta}\left[\mathscr{G}_{i}, B\right]\right]=\sqcap_{i \in \Delta}\left[f_{\varphi_{\psi}}^{-1}\left(\left[\mathscr{G}_{i}, B\right]\right)\right]$.

Definition 13. [16] A type-2 soft set $[\mathscr{E}, A] \neq[\widetilde{\phi}, A]$ over $(X, A)$ is called a

(i) type - 2 soft element of $X$, if $\mathscr{E}(\alpha)$ is a type-1 g-soft element or simply a soft element of $X, \forall \alpha \in A$.

(ii) constant type -2 soft element of $X$, if $\exists$ a g-soft element $(E, A)$ such that $\mathscr{E}(\alpha)=(E, A), \forall \alpha \in A$.

(iii) pseudo constant type-2 soft element of $X$, if $\exists$ a g-soft element $(E, A)$ such that $\mathscr{E}(\alpha)=(\widetilde{\phi}, A)$ or $(E, A), \forall \alpha \in A$.

We denotes $\mathscr{E}_{E}$, the constant type-2 soft element defined by $\mathscr{E}(\alpha)=(E, A), \forall \alpha \in A$ and $\mathscr{E}_{E}^{\alpha}$, the type-2 soft element defined by $\mathscr{E}(\alpha)=(E, A)$ and $\mathscr{E}(\beta)=(\widetilde{\phi}, A), \forall \beta(\neq \alpha) \in A$.

Definition 14. [16] The type-2 soft mapping $f_{\varphi_{\psi}}: S_{2}(X, A) \rightarrow S_{2}(Y, B)$ is called constant if $\exists$ a type-2 soft element $[\mathscr{E}, B] \in S_{2}(Y, B)$ such that $f_{\varphi_{\psi}}([\mathscr{F}, A])=[\mathscr{E}, B], \forall[\mathscr{F}, A][\neq(\widetilde{\phi}, A)] \in S_{2}(X, A)$.

Proposition 7. [16] The type-2 soft mapping $f_{\varphi_{\psi}}: S_{2}(X, A) \rightarrow S_{2}(Y, B)$ is constant iff the mappings $f: X \rightarrow Y, \varphi: A \rightarrow B$ and $\psi: A \rightarrow B$ are constant.

Definition 15. Let $[\mathscr{F}, A] \in S_{2}(X, A)$ and $[\mathscr{G}, B] \in S_{2}(Y, B)$. The Cartesian product of $[\mathscr{F}, A]$ and $[\mathscr{G}, B]$ is denoted by $[\mathscr{F}, A] \times[\mathscr{G}, B]=[\mathscr{F} \times \mathscr{G}, A \times B] \in S_{2}(X \times Y, A \times B)$ and defined as $\forall(\alpha, \beta) \in A \times B,[\mathscr{F} \times \mathscr{G}](\alpha, \beta)=\mathscr{F}(\alpha) \tilde{\times} \mathscr{G}(\beta)$.

Proposition 8. Let $[\mathscr{F}, A],\left[\mathscr{F}_{1}, A\right],\left[\mathscr{F}_{2}, A\right] \in S_{2}(X, A)$ and $[\mathscr{G}, B],\left[\mathscr{G}_{1}, B\right],\left[\mathscr{G}_{2}, B\right],[\mathscr{H}, B] \in S_{2}(Y, B)$. Then

(i) $[\mathscr{F}, A] \times[[\mathscr{G}, B] \cup[\mathscr{H}, B]]=[[\mathscr{F}, A] \times[\mathscr{G}, B]] \cup[[\mathscr{F}, A] \times[\mathscr{H}, B]]$;

(ii) $[\mathscr{F}, A] \times[[\mathscr{G}, B] \cap[\mathscr{H}, B]]=[[\mathscr{F}, A] \times[\mathscr{G}, B]] \cap[[\mathscr{F}, A] \times[\mathscr{H}, B]]$;

(iii) $\left[\left[\mathscr{F}_{1}, A\right] \times\left[\mathscr{G}_{1}, B\right]\right] \cap\left[\left[\mathscr{F}_{2}, A\right] \times\left[\mathscr{G}_{2}, B\right]\right]=\left[\left[\mathscr{F}_{1}, A\right] \cap\left[\mathscr{F}_{2}, A\right]\right] \times\left[\left[\mathscr{G}_{1}, B\right] \cap\left[\mathscr{G}_{2}, B\right]\right]$.

Proof. Proofs are follows from Proposition 5.

Definition 16. [5] Let $\tau$ be a family of soft sets over $(X, A)$. Define $\tau(e)=\{F(e): F \in \tau\}$ for $e \in A$. The $\tau$ is said to be a topology of soft subsets over $(X, A)$ if $\tau(e)$ is a crisp topology on $X, \forall e \in A$. 
Definition 17. [5] Let $(X, A)$ be the universe. Let $\mathbf{T}: A \rightarrow P\left(P(X)^{A}\right)$ be a soft set over $\left(P(X)^{A}, A\right)$. Now $\mathbf{T}$ is said to be a soft topology over $(X, A)$ iffor each $e \in A, \mathbf{T}(e)$ is a topology of soft subsets over $(X, A)$.

Definition 18. [17] A subcollection $\tau$ of $S_{1}(X, A)$ is said to be a soft topology on $X$ if the following prperties are satisfied:

(i) $(\widetilde{\Phi}, A),(\widetilde{X}, A) \in \tau$, where $\widetilde{\Phi}(\alpha)=\phi$ and $\widetilde{X}(\alpha)=X, \forall \alpha \in A$;

(ii) the intersection of any two soft sets in $\tau$ belongs to $\tau$;

(iii) the union of any number of soft sets in $\tau$ belongs to $\tau$.

Remark. If $\tau$ be a Naz type soft topology defined in Definition 18, then $\tau(\alpha)=\{F(\alpha):(F, A) \in \tau\}$ is a crisp topology on $X$ and hence $\tau$ is a topology of soft subsets, defined by Hazra et al. [5]. But the converse is not true in general which is shown in [12]. Also if $\tau$ be a topology of soft subsets, then we can define a topology of soft subsets $\tau^{*}$ which is a Naz type soft topology with $\tau(\alpha)=\tau^{*}(\alpha), \alpha \in A[12]$.

\section{Type-2 soft topologies}

In this section, we have introduced type-2 soft topology and study some of its important properties. Unless otherwise stated, let $X$ be a non empty set, $A$ be the set of parameters and $S_{2}(X, A)$ be the family of all type-2 soft sets over $(X, A)$.

Definition 19. A subcollection $\mathscr{T}$ of $S_{2}(X, A)$ is said to be a type-2 soft topology on $X$ if the following prperties are satisfied:

(i) $[\widetilde{\Phi}, A],[\widetilde{\mathscr{X}}, A] \in \mathscr{T}$, where $\widetilde{\Phi}(\alpha)=(\widetilde{\phi}, A)$ and $\widetilde{\mathscr{X}}(\alpha)=(\widetilde{X}, A), \forall \alpha \in A$;

(ii) the intersection of any two type-2 soft sets in $\mathscr{T}$ belongs to $\mathscr{T}$;

(iii) the union of any number of type-2 soft sets in $\mathscr{T}$ belongs to $\mathscr{T}$.

The triplet $[X, A, \mathscr{T}]$ is called a type-2 soft topological space over $X$.

Example 1. Let $X$ be a non-empty set and $A$ be a set of parameters. Then $\mathscr{T}_{1}=\{[\widetilde{\Phi}, A],[\widetilde{\mathscr{X}}, A]\}, \mathscr{T}_{2}=S_{2}(X, A)$ and $\mathscr{T}_{3}=\{[\widetilde{\Phi}, A],[\mathscr{F}, A],[\widetilde{\mathscr{X}}, A]\}$, where $[\mathscr{F}, A](\neq[\widetilde{\Phi}, A])$ be any type-2 soft set are the examples of type-2 soft topologies. $\mathscr{T}_{1}, \mathscr{T}_{2}$ are called type-2 indiscrete soft topology and type-2 discrete soft toplogy on $X$ respectively.

Proposition 9. Let $\mathscr{T}_{1}, \mathscr{T}_{2}$ be any two type-2 soft topologies on X. Then their intersection $\mathscr{T}_{1} \sqcap \mathscr{T}_{2}=\{[\mathscr{F}, A]:[\mathscr{F}, A] \in$ $\mathscr{T}_{1}$ and $\left.[\mathscr{F}, A] \in \mathscr{T}_{2}\right\}$ is a type-2 soft toplogy on $X$.

Proof. Proof is straightforward.

Remark. The union of any two type-2 soft topologies on $X$ may not be a type-2 soft topology on $X$ in general, which shows the following example.

Example 2. Let $X$ be a nonempty set, $Y$ is a proper subset of $X$ and $A=\{\alpha, \beta\}$. Let $[\mathscr{F} 1, A]=\{(\widetilde{Y}, A),(\widetilde{\phi}, A)\}$ and $\left[\mathscr{F}_{2}, A\right]=\{(\widetilde{\phi}, A),(\widetilde{Y}, A)\}$ be two type-2 soft set. Then $\mathscr{T}_{1}=\left\{[\widetilde{\Phi}, A],\left[\mathscr{F}_{1}, A\right],[\widetilde{\mathscr{X}}, A]\right\}, \mathscr{T}_{2}=\left\{[\widetilde{\Phi}, A],\left[\mathscr{F}_{2}, A\right],[\widetilde{\mathscr{X}}, A]\right\}$ are type-2 soft topologies on $X$ but their union $\mathscr{T}_{1} \sqcup \mathscr{T}_{2}=\left\{[\widetilde{\Phi}, A],\left[\mathscr{F}_{1}, A\right],\left[\mathscr{F}_{2}, A\right],[\widetilde{\mathscr{X}}, A]\right\}$ is not a type-2 soft topology on $X$.

Proposition 10. Let $[X, A, \mathscr{T}]$ be a type-2 soft topological space over $X$. Then for each $\alpha \in A$, the collection $\mathscr{T}^{\alpha}=$ $\{\mathscr{F}(\alpha):[\mathscr{F}, A] \in \mathscr{T}\}$ defines a soft topology of Shabir and Naz [17] on X.

Proof. Let $[X, A, \mathscr{T}]$ be a type-2 soft topological space over $X$ and $\alpha$ be any member of $A$. Since $[\widetilde{\Phi}, A],[\widetilde{\mathscr{X}}, A] \in \mathscr{T}$, it follows that $(\widetilde{\phi}, A),(\widetilde{X}, A) \in \mathscr{T}^{\alpha}$.

Again let $\left(F_{1}, A\right),\left(F_{2}, A\right)$ be any two members of $\mathscr{T}^{\alpha}$. Then $\exists$ two type-2 soft sets $\left[\mathscr{F}_{1}, A\right],\left[\mathscr{F}_{1}, A\right]$ in $\mathscr{T}$ such that 
$\mathscr{F}_{1}(\alpha)=\left(F_{1}, A\right)$ and $\mathscr{F}_{2}(\alpha)=\left(F_{2}, A\right)$. Thus $\left[\mathscr{F}_{1}, A\right] \sqcap\left[\mathscr{F}_{1}, A\right]=\left[\mathscr{F}_{1} \sqcap \mathscr{F}_{2}, A\right]$ is a member of $\mathscr{T}$ and hence, $\left(\mathscr{F}_{1} \sqcap \mathscr{F}_{2}\right)(\alpha)=\mathscr{F}_{1}(\alpha) \widetilde{\cap}_{\mathscr{F}_{2}}(\alpha)=\left(F_{1}, A\right) \widetilde{\cap}\left(F_{2}, A\right)$ is a member of $\mathscr{T}^{\alpha}$.

Next let $\left(F_{i}, A\right), i \in \Delta$ be any collection of members of $\mathscr{T}^{\alpha}$. Then $\exists$ type-2 soft sets $\left[\mathscr{F}_{i}, A\right], i \in \Delta$ in $\mathscr{T}$ such that $\mathscr{F}_{i}(\alpha)=\left(F_{i}, A\right), \quad i \in \Delta$. Thus $\sqcup_{i \in \Delta}\left[\mathscr{F}_{i}, A\right]=\left[\sqcup_{i \in \Delta} \mathscr{F}_{1}, A\right]$ is a member of $\mathscr{T}$ and hence, $\left(\sqcup_{i \in \Delta} \mathscr{F}_{i}\right)(\alpha)=\widetilde{\cup}_{i \in \Delta} \mathscr{F}_{1}(\alpha)=\widetilde{\cup}_{i \in \Delta}\left(F_{i}, A\right)$ is a member of $\mathscr{T}^{\alpha}$. Therefore, $\mathscr{T}^{\alpha}$ is a soft topology of Shabir and Naz over $X, \forall \alpha \in A$.

Remark. From Proposition 10, it can be seen that if $[X, A, \mathscr{T}]$ is a type-2 soft topological space over $X$, then there is a mapping $\alpha \rightarrow \mathscr{T}(\alpha)$ from the parameter set $A$ to the collection of all soft topologies on $(X, A)$. Thus, the question is whether the converse of the above is true or not. In the next Proposition, we get an affirmative answer to this question, and a relation between the two representations of the type- 2 soft topology is obtained.

Proposition 11. Let $X$ be a non empty set and $A$ be a set of parameters. If $\mathscr{T}: A \rightarrow P\left(S_{1}(X, A)\right)$ be a mapping defined by $\mathscr{T}(\alpha)$ is a soft topology over $X, \forall \alpha \in A$, then $\exists$ a type-2 soft topology $\mathscr{T}^{*}$ over $X$ such that $\left[\mathscr{T}^{*}\right]^{\alpha}=\mathscr{T}(\alpha), \forall \alpha \in A$.

Proof. Define $\quad \mathscr{T}^{*}=\left\{[\mathscr{F}, A] \in S_{2}(X, A): \mathscr{F}^{*}(\alpha) \in \mathscr{T}(\alpha), \quad \forall \quad \alpha \quad \in \quad A\right\}$. Since $\widetilde{\Phi}(\alpha)=(\widetilde{\phi}, A) \in \mathscr{T}(\alpha), \forall \alpha \in A \Rightarrow[\widetilde{\Phi}, A] \in \mathscr{T}^{*}$ and $\widetilde{\mathscr{X}}(\alpha)=(\widetilde{X}, A) \in \mathscr{T}(\alpha), \forall \alpha \in A \Rightarrow[\widetilde{\mathscr{X}}, A] \in \mathscr{T}^{*}$.

Again let $\left[\mathscr{F}_{1}, A\right],\left[\mathscr{F}_{2}, A\right] \in \mathscr{T}^{*}$. Then $\mathscr{F}_{1}(\alpha), \mathscr{F}_{2}(\alpha) \in \mathscr{T}(\alpha), \forall \alpha \in A$.

Thus, $\mathscr{F}_{1}(\alpha) \widetilde{\cap}_{\mathscr{F}_{2}}(\alpha)=\left[\mathscr{F}_{1} \sqcap \mathscr{F}_{2}\right](\alpha) \in \mathscr{T}(\alpha), \forall \alpha \in A$.

Therefore, $\left[\mathscr{F}_{1}, A\right] \sqcap\left[\mathscr{F}_{2}, A\right]=\left[\mathscr{F}_{1} \sqcap \mathscr{F}_{2}, A\right] \in \mathscr{T}^{*}$.

Also let $\left[\mathscr{F}_{i}, A\right] \in \mathscr{T}^{*}, \quad \forall i \in \Delta . \quad$ Then $\mathscr{F}_{i}(\alpha) \in \mathscr{T}(\alpha), \quad \forall \quad \alpha \in A, \quad \forall \quad i \in \Delta$. Thus, $\widetilde{\cup}_{i \in \Delta} \mathscr{F}_{i}(\alpha)=\left[\sqcup_{i \in \Delta} \mathscr{F}_{i}\right](\alpha) \in \mathscr{T}(\alpha), \forall \alpha \in A$. Therefore, $\left[\sqcup_{i \in \Delta} \mathscr{F}_{i}, A\right]=\sqcup_{i \in \Delta}\left[\mathscr{F}_{i}, A\right] \in \mathscr{T}^{*}$. Therefore $\mathscr{T}^{*}$ is a type-2 soft topology on $X$.

Next let $(U, A) \in \mathscr{T}(\alpha)$. Now we can define a type-2 soft set $[\mathscr{F}, A]$ by $\mathscr{F}(\alpha)=(U, A)$ and $\mathscr{F}(\beta)=(\phi, A) \forall \beta \neq \alpha$. Then $[\mathscr{F}, A] \in \mathscr{T}^{*}$ and $(U, A)=\mathscr{F}(\alpha) \in\left[\mathscr{T}^{*}\right]^{\alpha}$. Therefore, $\mathscr{T}(\alpha) \subseteq\left[\mathscr{T}^{*}\right]^{\alpha} \ldots \ldots \ldots .(1)$. Also let $(V, A) \in\left[\mathscr{T}^{*}\right]^{\alpha}$. Then $\exists[\mathscr{F}, A] \in \mathscr{T}^{*}$ such that $(V, A)=\mathscr{F}(\alpha) \in \mathscr{T}(\alpha)$. Therefore $\left[\mathscr{T}^{*}\right]^{\alpha} \subseteq \mathscr{T}(\alpha) \ldots \ldots . . .(2)$. Therefore, from (1) and (2) we get $\mathscr{T}(\alpha)=\left[\mathscr{T}^{*}\right]^{\alpha}, \forall \alpha \in A$.

Remark. If $\mathscr{T}$ be a type-2 soft topology on $X$ and $\mathscr{T}^{*}$ be the type-2 soft topology on $X$ constructed by using Proposition 11. Then these two type-2 soft topologies may be different, which is shown by the following example.

Example 3. Let $X$ be a non-empty set and $A=\{\alpha, \beta\}$. Let $\mathscr{T}=\{[\widetilde{\Phi}, A],[\widetilde{\mathscr{X}}, A]\}$ be a type-2 soft topology on $X$. Then $\mathscr{T}^{\alpha}=\mathscr{T}^{\beta}=\{(\widetilde{\phi}, A),(\widetilde{X}, A)\}$.

Let $\left[\mathscr{F}_{1}, A\right]=\{(\widetilde{X}, A) / \alpha, \quad(\widetilde{\phi}, A) / \beta\} \quad$ and $\quad\left[\mathscr{F}_{2}, A\right]=\{(\widetilde{\phi}, A) / \alpha, \quad(\widetilde{X}, A) / \beta\} . \quad$ Thus $\mathscr{T}^{*}=\left\{[\widetilde{\Phi}, A],\left[\mathscr{F}_{1}, A\right],\left[\mathscr{F}_{2}, A\right],[\widetilde{\mathscr{X}}, A]\right\}$. So, $\mathscr{T}^{*}$ is different from $\mathscr{T}$ but $\left[\mathscr{T}^{*}\right]^{\alpha}=[\mathscr{T}]^{\alpha}, \forall \alpha \in A$.

Remark. If $\mathscr{T}$ be a type-2 soft topology, then from Proposition 10, it can be seen that $\mathscr{T}$ is a Hazra et al. type soft topology on $X$. But the converse is not true in general which is shown by the following example. Also for any Hazra et al. type soft topology $\mathbf{T}$, we can constract a Hazra et al. type soft topology $\mathbf{T}^{*}$ which is a type- 2 soft topology and for all $\alpha \in A,\left[\mathbf{T}^{*}(\alpha)\right](\beta)=[\mathbf{T}(\alpha](\beta), \forall \beta \in A$.

Example 4. Let $X$ be a non-empty set and $A=\{\alpha, \beta\}$. Define a soft set $\mathbf{T}: A \rightarrow P\left(P(X)^{A}\right)$ by $\mathbf{T}(\alpha)=\{(F, A),(G, A)\}, \mathbf{T}(\beta)=\{(F, A),(G, A)\}$, where $F(\alpha)=\phi, F(\beta)=X, G(\alpha)=X, G(\beta)=\phi$. Then $\mathbf{T}$ is a Hazra et al. type soft topology on $X$, but $\mathbf{T}$ is not a type-2 soft topology on $X$ since $\mathbf{T}(\alpha)=\{(F, A),(G, A)\}$ is not a Naz 
type soft topology on $X$.

Again we define a soft set $\mathbf{T}^{*}: A \rightarrow P\left(P(X)^{A}\right)$ by $\mathbf{T}^{*}(\alpha)=\{(F, A): F(\beta) \in[\mathbf{T}(\alpha)](\beta), \forall \beta \in A\}, \forall \alpha \in A$. Then $\mathbf{T}^{*}$ is a Hazra et al. type soft topology as well as a type-2 soft topology and for all $\alpha \in A,\left[\mathbf{T}^{*}(\alpha)\right](\beta)=[\mathbf{T}(\alpha](\beta), \forall \beta \in A$. of Hazra et al.,a type-2 soft topology and $\left[\mathbf{T}^{*}(\alpha)\right](\beta)=[\mathbf{T}(\alpha](\beta), \forall \alpha, \beta \in A$.

Definition 20. Let $X$ and $Y$ be two non-empty sets, $\mathscr{T}, \mathscr{U}$ be two type-2 soft topologies on $X, Y$ respectively and $f_{\varphi_{\psi}}:[X, A, \mathscr{T}] \rightarrow[Y, B, \mathscr{U}]$ be a type-2 soft mapping. The image of $\mathscr{T}$ and the preimage of $\mathscr{U}$ under $f_{\varphi_{\psi}}$ are denoted by $f_{\varphi_{\psi}}(\mathscr{T})$ and $f_{\varphi_{\psi}}^{-1}(\mathscr{U})$ respectively, defined by

(i) $f_{\varphi_{\psi}}(\mathscr{T})=\left\{[\mathscr{G}, B] \in S_{2}(Y, B): f_{\varphi_{\psi}}^{-1}([\mathscr{G}, B])=\left[f_{\varphi_{\psi}}^{-1}(\mathscr{G}), A\right] \in \mathscr{T}\right\}$ and

(ii) $f_{\varphi_{\psi}}^{-1}(\mathscr{U})=\left\{f_{\varphi_{\psi}}^{-1}([\mathscr{G}, B])=\left[f_{\varphi_{\psi}}^{-1}(\mathscr{G}), A\right]:[\mathscr{G}, B] \in \mathscr{U}\right\}$.

Proposition 12. Let $X$ and $Y$ be two non-empty sets, $\mathscr{T}, \mathscr{U}$ be two type-2 soft topologies on $X, Y$ respectively and $f_{\varphi_{\Psi}}:[X, A, \mathscr{T}] \rightarrow[Y, B, \mathscr{U}]$ be a type-2 soft mapping. Then

(i) $f_{\varphi_{\psi}}^{-1}(\mathscr{U})$ is a type-2 soft topology on $X$, and

(ii) $f_{\varphi_{\psi}}(\mathscr{T})$ is a type-2 soft topology on $Y$.

Proof. Proofs follows from Proposition 6.

Proposition 13. Let $[X, A, \mathscr{T}]$ be a type-2 soft topological space and $G \subseteq X$. Then $\mathscr{T}_{G}=\{[\mathscr{F}, A] \sqcap[\widetilde{\mathscr{G}}, A]:[\mathscr{F}, A] \in \mathscr{T}\}$, where $\widetilde{\mathscr{G}}(\alpha)=(\widetilde{G}, A), \forall \alpha \in A$ is a type-2 soft topology on $G$.

Proof. (i) Since $[\widetilde{\Phi}, A]$ and $[\widetilde{\mathscr{X}}, A] \in \mathscr{T} \Rightarrow[\widetilde{\Phi}, A]$ and $[\widetilde{\mathscr{G}}, A] \in \mathscr{T}_{G}$.

(ii) Let $\left[\mathscr{H}_{1}, A\right],\left[\mathscr{H}_{2}, A\right] \in \mathscr{T}_{G}$.

Then $\exists\left[\mathscr{F}_{1}, A\right],\left[\mathscr{F}_{2}, A\right] \in \mathscr{T}$ such that $\left[\mathscr{H}_{1}, A\right]=\left[\mathscr{F}_{1}, A\right] \sqcap\left[\widetilde{\mathscr{G}}_{, A}\right]$ and $\left[\mathscr{H}_{2}, A\right]=\left[\mathscr{F}_{2}, A\right] \sqcap[\widetilde{\mathscr{G}}, A]$.

Since $\mathscr{T}$ is a type-2 soft topology, it follows that $\left[\mathscr{F}_{1}, A\right] \sqcap\left[\mathscr{F}_{2}, A\right] \in \mathscr{T}$ and $\left[\mathscr{H}_{1}, A\right] \sqcap\left[\mathscr{H}_{2}, A\right]=\left[\mathscr{F}_{1}, A\right] \sqcap\left[\mathscr{F}_{2}, A\right] \sqcap$ $[\widetilde{\mathscr{G}}, A] \in \mathscr{T}_{G}$.

(iii) Next let $\left[\mathscr{H}_{i}, A\right] \in \mathscr{T}_{G}, i \in \Delta$.

Then $\exists\left[\mathscr{F}_{i}, A\right] \in \mathscr{T}$ such that $\left[\mathscr{H}_{i}, A\right]=\left[\mathscr{F}_{i}, A\right] \sqcap\left[\widetilde{\mathscr{G}}_{,} A\right], i \in \Delta$.

Since $\mathscr{T}$ is a type-2 soft topology, it implies that $\bigsqcup_{i \in \Delta}\left[\mathscr{F}_{i}, A\right] \in \mathscr{T}$ and

$\bigsqcup_{i \in \Delta}\left[\mathscr{H}_{i}, A\right]=\bigsqcup_{i \in \Delta}\left(\left[\mathscr{F}_{i}, A\right] \sqcap\left[\widetilde{\mathscr{G}}_{, A}\right]\right)=\left[\bigsqcup_{i \in \Delta}\left[\mathscr{F}_{i}, A\right]\right] \sqcap[\widetilde{\mathscr{G}}, A] \in \mathscr{T}_{G}$.

Therefore $\mathscr{T}_{G}$ is a type-2 soft topology on $G$.

Definition 21. Let $[X, A, \mathscr{T}]$ and $[Y, B, \mathscr{U}]$ be two type-2 soft topological spaces. The type-2 soft mapping $f_{\varphi_{\Psi}}:[X, A, \mathscr{T}] \rightarrow[Y, A, \mathscr{U}]$ is said to be

(i) type-2 soft continuous if $f_{\varphi_{\psi}}^{-1}([\mathscr{G}, B]) \in \mathscr{T}, \forall[\mathscr{G}, B] \in \mathscr{U}$.

(ii) type-2 soft open if $f_{\varphi_{\psi}}([\mathscr{F}, A]) \in \mathscr{U}, \forall[\mathscr{F}, A] \in \mathscr{T}$.

Proposition 14. Let $[X, A, \mathscr{T}]$ and $[Y, B, \mathscr{U}]$ be two type-2 soft topological spaces. A type-2 soft mapping $f_{\varphi_{\psi}}:[X, A, \mathscr{T}] \rightarrow[Y, B, \mathscr{U}]$ is type-2 soft continuous iff $\forall$ type-2 soft element $[\mathscr{E}, A]$ of $X$ and $\forall[\mathscr{G}, B] \in \mathscr{U}$ such that $f_{\varphi_{\psi}}([\mathscr{E}, A]) \sqsubseteq[\mathscr{G}, B], \exists[\mathscr{F}, A] \in \mathscr{T}$ such that $[\mathscr{E}, A] \sqsubseteq[\mathscr{F}, A]$ and $f_{\varphi_{\psi}}([\mathscr{F}, A]) \sqsubseteq[\mathscr{G}, B]$.

Proof. Let $f_{\varphi_{\psi}}:[X, A, \mathscr{T}] \rightarrow[Y, B, \mathscr{U}]$ be type-2 soft continuous. Let $[\mathscr{E}, A]$ be any type-2 soft element in $X$ and $[\mathscr{G}, B] \in \mathscr{U}$ such that $f_{\varphi_{\psi}}([\mathscr{E}, A]) \sqsubseteq[\mathscr{G}, B]$. Then $\left[f_{\varphi_{\psi}}([\mathscr{E}, A])\right](\beta) \widetilde{\subseteq} \mathscr{G}(\beta), \forall \beta \in B$.

Since $f_{\varphi_{\psi}}$ is type-2 soft continuous, it follows that $f_{\varphi_{\psi}}^{-1}([\mathscr{G}, B])=\left[f_{\varphi_{\psi}}^{-1}(\mathscr{G}), A\right] \in \mathscr{T}$. Let $[\mathscr{F}, A]=\left[f_{\varphi_{\psi}}^{-1}(\mathscr{G}), A\right]$. Now for each $\alpha \in A$,

$$
\begin{aligned}
\mathscr{F}(\alpha) & =\left[f_{\varphi_{\psi}}^{-1}(\mathscr{G})\right](\alpha)=f_{\varphi}^{-1}[\mathscr{G}(\psi(\alpha))] \widetilde{\supseteq} f_{\varphi}^{-1}\left[\left[f_{\varphi_{\psi}}([\mathscr{E}, A])\right](\psi(\alpha))\right] \\
& =f_{\varphi_{\psi}}^{-1}\left[\left[f_{\varphi_{\psi}}([\mathscr{E}, A])\right](\alpha)\right]=\left[f_{\varphi_{\psi}}^{-1}\left[f_{\varphi_{\psi}}([\mathscr{E}, A])\right]\right](\alpha) \supseteq[\mathscr{E}, A](\alpha)
\end{aligned}
$$


Thus $[\mathscr{E}, A] \sqsubseteq[\mathscr{F}, A]$ and $f_{\varphi_{\psi}}([\mathscr{F}, A])=f_{\varphi_{\psi}}\left(f_{\varphi_{\psi}}^{-1}([\mathscr{G}, B])\right) \sqsubseteq[\mathscr{G}, B]$. Therefore the given condition is satisfied.

Conversely let the given condition be satisfied.

Let $[\mathscr{G}, B] \in \mathscr{U}$. We are to show that $f_{\varphi_{\psi}}^{-1}([\mathscr{G}, B]) \in \mathscr{T}$. Let $[\mathscr{E}, A]$ be any type-2 soft element of $X$ such that $[\mathscr{E}, A] \sqsubseteq f_{\varphi_{\psi}}^{-1}([\mathscr{G}, B])$. Then $f_{\varphi_{\psi}}([\mathscr{E}, A]) \sqsubseteq f_{\varphi_{\psi}}\left[f_{\varphi_{\psi}}^{-1}\left(\left[\mathscr{G}_{, B}, B\right] \sqsubseteq[\mathscr{G}, B]\right.\right.$. So by the given condition $\exists\left[\mathscr{F}_{\mathscr{E}}, A\right] \in \mathscr{T}$ such that $[\mathscr{E}, A] \sqsubseteq\left[\mathscr{F}_{\mathscr{E}}, A\right]$ and $f_{\varphi_{\psi}}\left(\left[\mathscr{F}_{\mathscr{E}}, A\right]\right) \sqsubseteq\left[\mathscr{G}_{, B}\right]$ i.e. $\left[\mathscr{F}_{\mathscr{E}}, A\right] \sqsubseteq f_{\varphi_{\psi}}^{-1}\left[f_{\varphi_{\psi}}\left(\left[\mathscr{F}_{\mathscr{E}}, A\right]\right)\right] \sqsubseteq f_{\varphi_{\psi}}^{-1}\left(\left[\mathscr{G}_{,} B\right]\right)$. Now

$$
\begin{aligned}
f_{\varphi_{\psi}}^{-1}([\mathscr{G}, B]) & =\sqcup\left\{[\mathscr{E}, A]:[\mathscr{E}, A] \text { be any type-2 soft element of } X \text { such that }[\mathscr{E}, A] \sqsubseteq f_{\varphi_{\psi}}^{-1}([\mathscr{G}, B])\right\} \\
& \sqsubseteq \sqcup\left\{\left[\mathscr{F}_{\mathscr{E}}, A\right]:[\mathscr{E}, A] \text { be any type-2 soft element of } X \text { such that }[\mathscr{E}, A] \sqsubseteq f_{\varphi_{\psi}}^{-1}([\mathscr{G}, B])\right\} \\
& \sqsubseteq f_{\varphi_{\psi}}^{-1}([\mathscr{G}, B]) .
\end{aligned}
$$

Thus $f_{\varphi_{\psi}}^{-1}\left(\left[\mathscr{G}_{, B}\right]\right)=\sqcup\left\{\left[\mathscr{F}_{\mathscr{E}}, A\right]\right\}$ where each $\left[\mathscr{F}_{\mathscr{E}}, A\right] \in \mathscr{T}$ and hence, $f_{\varphi_{\psi}}^{-1}([\mathscr{G}, B]) \in \mathscr{T}$. Therefore $f_{\varphi_{\psi}}$ is type-2 soft continuous.

Proposition 15. Let $[X, A, \mathscr{T}]$ be a type-2 soft topological space. Then the identity type-2 soft mapping $f_{\varphi_{\Psi}}:[X, A, \mathscr{T}] \rightarrow$ $[X, A, \mathscr{T}]$ is type-2 soft continuous.

Proof. Since $f_{\varphi_{\psi}}$ is an identity type-2 soft mapping, it follows that $f_{\varphi}$ and $\psi$ are identity mappings. Let $[\mathscr{F}, A] \in \mathscr{T}$. Then, $\left[f_{\varphi_{\psi}}^{-1}([\mathscr{F}, A])\right](\alpha)=f_{\varphi}^{-1}[\mathscr{F}(\psi(\alpha))]=f_{\varphi}^{-1}[\mathscr{F}(\alpha)]=\mathscr{F}(\alpha), \forall \alpha \in A$. Thus, $f_{\varphi_{\psi}}^{-1}([\mathscr{F}, A])=[\mathscr{F}, A]$. Therefore, the identity type-2 soft mapping $f_{\varphi_{\psi}}:[X, A, \mathscr{T}] \rightarrow[X, A, \mathscr{T}]$ is type-2 soft continuous.

Proposition 16. Let $[X, A, \mathscr{T}]$ and $[Y, B, \mathscr{U}]$ be two type-2 soft topological spaces. If $\mathscr{T}$ contains all those type-2 soft sets of the form $\left[\widetilde{\left(\widetilde{X_{0}}, A\right)}, A\right]$ where $X_{0}$ be any subset of $X$, then the constant type-2 soft mapping $f_{\varphi_{\psi}}:[X, A, \mathscr{T}] \rightarrow[Y, B, \mathscr{U}]$ is type-2 soft continuous.

Proof. Since $f_{\varphi_{\psi}}:[X, A, \mathscr{T}] \rightarrow[Y, B, \mathscr{U}]$ is a constant type-2 soft mapping, it follows that the mappings $f: X \rightarrow Y, \psi$ : $A \rightarrow B$ and $\varphi: A \rightarrow B$ are constant. Let $\psi(\alpha)=\beta^{\prime}$ and $\varphi(\alpha)=\beta^{\prime \prime} \forall \alpha \in A$. Let $[\mathscr{G}, B]$ be any member of $\mathscr{U}$. Then for any $\alpha \in A$,

$$
\left[f_{\varphi_{\psi}}^{-1}([\mathscr{G}, B])(\alpha)=f_{\varphi}^{-1}[\mathscr{G}[\psi(\alpha)]]=f_{\varphi}^{-1}\left[\mathscr{G}\left(\beta^{\prime}\right)\right]=\left\{\begin{array}{l}
(\widetilde{\phi}, A), \forall \alpha \in A \text { if }\left[\mathscr{G}\left(\beta^{\prime}\right)\right]\left(\beta^{\prime \prime}\right)=\phi \\
\left(\widetilde{X_{0}}, A\right), \text { where } X_{0} \subseteq X, \forall \alpha \in A \text { if }\left[\mathscr{G}\left(\beta^{\prime}\right)\right]\left(\beta^{\prime \prime}\right) \neq \phi
\end{array}\right.\right.
$$

Hence, $f_{\varphi_{\psi}}^{-1}([\mathscr{G}, B])=[\widetilde{\Phi}, A]$ or $\left[\widetilde{\left(\widetilde{X_{0}}, A\right)}, A\right]$. Since $[\widetilde{\Phi}, A],\left[\widetilde{\left(\widetilde{X_{0}}, A\right)}, A\right] \in \mathscr{T}$, it follows that the constant type-2 soft mapping $f_{\varphi_{\Psi}}:[X, A, \mathscr{T}] \rightarrow[Y, B, \mathscr{U}]$ is type-2 soft continuous.

Proposition 17. Let $[X, A, \mathscr{T}],[Y, B, \mathscr{U}]$ and $[Z, C, \mathscr{W}]$ be type-2 soft topological spaces. If $f_{\varphi_{\Psi}}:[X, A, \mathscr{T}] \rightarrow[Y, B, \mathscr{U}]$ and $g_{\xi_{\eta}}:[Y, B, \mathscr{U}] \rightarrow[Z, C, \mathscr{W}]$ are type-2 soft continuous, then the soft mapping $g_{\xi_{\eta}} \circ f_{\varphi_{\psi}}=(g f)_{\xi \varphi_{\eta \psi}}:[X, A, \mathscr{T}] \rightarrow[Z, C, \mathscr{W}]$ is type-2 soft continuous.

Proof. Proof is straightforward.

Definition 22. Let $[X, A, \mathscr{T}]$ be a type-2 soft topological space. A subcollection $\mathbb{B}$ of $\mathscr{T}$ is said to be an open base for $\mathscr{T}$ if every member of $\mathscr{T}$ can be expressed as the union of some members of $\mathbb{B}$.

Proposition 18. Let $[X, A, \mathscr{T}]$ and $[Y, B, \mathscr{U}]$ be type-2 soft topological spaces. Then $\mathbb{F}=\{[\mathscr{F}, A] \times[\mathscr{G}, B]:[\mathscr{F}, A] \in$ $\mathscr{T},[\mathscr{G}, B] \in \mathscr{U}\}$ forms an open base of a type-2 soft topology on $X \times Y$.

Proof. We note that $[\widetilde{\Phi}, A \times B]=[\widetilde{\mathscr{X}}, A] \times[\widetilde{\Phi}, B]=[\widetilde{\Phi}, A] \times[\widetilde{\mathscr{Y}}, B]$. Since $[\widetilde{\Phi}, A],[\widetilde{\mathscr{X}}, A] \in \mathscr{T}$ and $[\widetilde{\Phi}, B]$, $[\widetilde{\mathscr{Y}}, B] \in$ $\mathscr{U}$, it follows that $[\widetilde{\Phi}, A \times B],[\widetilde{\mathscr{X} \times \mathscr{Y}}, A \times B] \in \mathbb{F}$. Again let $\left[\mathscr{F}_{1}, A\right] \times\left[\mathscr{G}_{1}, B\right],\left[\mathscr{F}_{2}, A\right] \times\left[\mathscr{G}_{2}, B\right]$ be any two member 
of $\mathbb{F}$, where $\left[\mathscr{F}_{1}, A\right],\left[\mathscr{F}_{2}, A\right] \in \mathscr{T}$ and $\left[\mathscr{G}_{1}, B\right],\left[\mathscr{G}_{2}, B\right] \in \mathscr{U}$. So $\left[\mathscr{F}_{1}, A\right] \sqcap\left[\mathscr{F}_{2}, A\right] \in \mathscr{T}$ and $\left[\mathscr{G}_{1}, B\right] \sqcap\left[\mathscr{G}_{2}, B\right] \in \mathscr{U}$. Thus $\left[\left[\mathscr{F}_{1}, A\right] \times\left[\mathscr{G}_{1}, B\right]\right] \sqcap\left[\left[\mathscr{F}_{2}, A\right] \times\left[\mathscr{G}_{2}, B\right]\right]=\left[\left[\mathscr{F}_{1}, A\right] \sqcap\left[\mathscr{F}_{2}, A\right]\right] \times\left[\left[\mathscr{G}_{1}, B\right] \sqcap\left[\mathscr{G}_{2}, A\right]\right] \in \mathbb{F}$. Therefore $\mathbb{F}$ forms an open base of the type-2 soft topology on $X \times Y$.

Definition 23. The type-2 soft topology in $X \times Y$ induced by the open base $\mathbb{F}$ is said to be the product type-2 soft topology of the type-2 soft topologies $\mathscr{T}$ and $\mathscr{U}$. It is denoted by $\mathscr{T} \times \mathscr{U}$. The type-2 soft topological space $[X \times Y, A \times B, \mathscr{T} \times \mathscr{U}]$ is said to be the type-2 soft topological product of the type-2 soft topological spaces $[X, A, \mathscr{T}]$ and $[Y, B, \mathscr{U}]$.

Proposition 19. Let $[X, A, \mathscr{T}]$ and $[Y, B, \mathscr{U}]$ be two type-2 soft topological spaces. Then the projection soft mappings $\left[\Pi^{X}\right]_{\varphi_{\psi^{A}}^{A}}:[X \times Y, A \times B, \mathscr{T} \times \mathscr{U}] \rightarrow[X, A, \mathscr{T}]$ and $\left[\Pi^{Y}\right]_{\varphi_{\psi^{B}}^{B}}:[X \times Y, A \times B, \mathscr{T} \times \mathscr{U}] \rightarrow[Y, B, \mathscr{U}]$ are type-2 soft continuous and type-2 soft open where $\Pi^{X}: X \times Y \rightarrow X, \varphi^{A}: A \times B \rightarrow B$ and $\psi^{A}: A \times B \rightarrow A$ are mappings defined by $\Pi^{X}(x, y)=$ $x, \varphi^{A}(\alpha, \beta)=\alpha$ and $\psi^{A}(\alpha, \beta)=\alpha$. Also $\mathscr{T} \times \mathscr{U}$ is the smallest type-2 soft topology in $X \times Y$ for which the projection soft mappings are type-2 soft continuous.

Proof. $[\mathscr{F}, A] \in \mathscr{T} \Rightarrow\left[\Pi^{X}\right]_{\varphi_{\psi^{A}}^{A}}^{-1}([\mathscr{F}, A])=[\mathscr{F}, A] \times[\widetilde{\mathscr{Y}}, B]$ is a basic open set in $\mathscr{T} \times \mathscr{U}$. So $\Pi_{\varphi_{\psi^{A}}^{A}}^{X}$ is type-2 soft continuous.

Again let $[\mathscr{F}, A \times B] \in \mathscr{T} \times \mathscr{U}$. Then there exists a subfamily $\mathbb{F}^{\prime}$ of $\mathbb{F}$ such that $[\mathscr{F}, A \times B]$ is the union of the members of $\mathbb{F}^{\prime}$. So,

$$
\begin{aligned}
\Pi_{\varphi_{\psi^{A}}^{A}}^{X}([\mathscr{F}, A \times B]) & =\sqcup\left\{\Pi_{\varphi_{\psi^{A}}^{A}}^{X}[[\mathscr{G}, A] \times[\mathscr{H}, B]]:[\mathscr{G}, A] \times[\mathscr{H}, B] \in \mathbb{F}^{\prime}\right\} \\
& =\sqcup\left\{[\mathscr{G}, A]:[\mathscr{G}, A] \times[\mathscr{H}, B] \in \mathbb{F}^{\prime}\right\} \in \mathscr{T}(\text { since }[\mathscr{G}, A] \in \mathscr{T}) .
\end{aligned}
$$

Therefore $\pi_{\varphi_{\psi^{A}}^{A}}^{X}$ is type-2 soft open.

Similarly it can be shown that $\pi_{\varphi_{\psi^{B}}^{B}}^{Y}$ is also type-2 soft continuous and type-2 soft open.

Next let $\mathscr{W}$ be any type-2 soft topology on $X \times Y$ such that the soft mappings

$\left[\Pi^{X}\right]_{\varphi_{\psi^{A}}^{A}}:[X \times Y, A \times B, \mathscr{T} \times \mathscr{U}] \rightarrow[X, A, \mathscr{T}]$ and $\left[\Pi^{Y}\right]_{\varphi_{\psi^{B}}^{B}}:[X \times Y, A \times B, \mathscr{T} \times \mathscr{U}] \rightarrow[Y, B, \mathscr{U}]$ are type-2 soft continuous.

Let $[[\mathscr{F}, A] \times[\mathscr{G}, B]]$ be any basic open type-2 soft set in $\mathscr{T} \times \mathscr{U}$. Now,

$$
\begin{aligned}
{[[\mathscr{F}, A] \times[\mathscr{G}, B]] } & =[[\mathscr{F}, A] \sqcap[\widetilde{\mathscr{X}}, A]] \times[[\widetilde{\mathscr{Y}}, B] \sqcap[\mathscr{G}, B]] \\
& =[[\mathscr{F}, A] \times[\widetilde{\mathscr{Y}}, B]] \sqcap[[\widetilde{\mathscr{X}}, A] \times[\mathscr{G}, B]] \\
& =\left(\Pi_{\varphi_{\psi^{A}}^{A}}^{X}\right)^{-1}[[\mathscr{F}, A]] \sqcap\left(\Pi_{\varphi_{\psi^{B}}^{B}}^{Y}\right)^{-1}[[\mathscr{G}, B]] \in \mathscr{W} \\
& \left(\operatorname{since}\left(\Pi_{\varphi_{\psi^{A}}^{A}}^{X}\right)^{-1}[[\mathscr{F}, A]],\left(\Pi_{\varphi_{\psi^{B}}^{B}}^{Y}\right)^{-1}[[\mathscr{G}, B]] \in \mathscr{W}\right) .
\end{aligned}
$$

Thus $\mathscr{T} \times \mathscr{U}$ is a subcollection of $\mathscr{W}$. Therefore $\mathscr{T} \times \mathscr{U}$ is the smallest type-2 soft topology in $X \times Y$ for which the projection soft mappings are type-2 soft continuous.

Proposition 20. Let $[X \times Y, A \times B, \mathscr{T} \times \mathscr{U}]$ be the product space of two type-2 soft topological spaces $[X, A, \mathscr{T}]$ and $[Y, B, \mathscr{U}]$ and $\Pi_{\varphi_{\psi^{A}}^{A}}^{X}:[X \times Y, A \times B, \mathscr{T} \times \mathscr{U}] \rightarrow[X, A, \mathscr{T}]$ and $\pi_{\varphi_{\psi^{B}}^{B}}^{B}:[X \times Y, A \times B, \mathscr{T} \times \mathscr{U}] \rightarrow[Y, B, \mathscr{U}]$ be the soft projection mappings. If $[Z, C, \mathscr{W}]$ be any type-2 soft topological space, then the soft mapping $f_{\varphi_{\psi}}:[Z, C, \mathscr{W}] \rightarrow[X \times Y, A \times B, \mathscr{T} \times \mathscr{U}]$ is soft type-2 continuous iff the soft mappings $\pi_{\varphi_{\psi^{A}}^{A}}^{X} \circ f_{\varphi_{\psi}}:[Z, C, \mathscr{W}] \rightarrow[X, A, \mathscr{T}]$ and $\pi_{\varphi_{\psi^{B}}^{Y}}^{Y} \circ f_{\varphi_{\psi}}:[Z, C, \mathscr{W}] \rightarrow[Y, B, \mathscr{U}]$ are type-2 soft continuous. 
Proof. First let the soft mapping $f_{\varphi_{\Psi}}:[Z, C, \mathscr{W}] \rightarrow[X \times Y, A \times B, \mathscr{T} \times \mathscr{U}]$ be type-2 soft continuous. Also the soft mappings $\Pi_{\varphi_{\psi^{A}}^{A}}^{X}:[X \times Y, A \times B, \mathscr{T} \times \mathscr{U}] \rightarrow[X, A, \mathscr{T}]$ and $\pi_{\varphi_{\psi^{B}}^{B}}^{B}:[X \times Y, A \times B, \mathscr{T} \times \mathscr{U}] \rightarrow[Y, B, \mathscr{U}]$ are type-2 soft continuous.Then by Proposition 17, we have the composition soft mappings $\pi_{\varphi_{\psi^{A}}^{A}}^{X} \circ f_{\varphi_{\psi}}:[Z, C, \mathscr{W}] \rightarrow[X, A, \mathscr{T}]$ and $\pi_{\varphi_{\psi^{B}}^{B}}^{Y} \circ f_{\varphi_{\psi}}:[Z, C, \mathscr{W}] \rightarrow[Y, B, \mathscr{U}]$ are type-2 soft continuous.

Conversely let the soft mappings $\quad \Pi_{\varphi_{\psi^{A}}^{A}}^{X}:[X \times Y, A \times B, \mathscr{T} \times \mathscr{U}] \rightarrow[X, A, \mathscr{T}] \quad$ and $\pi_{\varphi_{\psi^{B}}^{B}}^{B}:[X \times Y, A \times B, \mathscr{T} \times \mathscr{U}] \rightarrow[Y, B, \mathscr{U}]$ are type-2 soft continuous. Let $[\mathscr{F}, A \times B] \in(\mathscr{T} \times \mathscr{U})$ and $\mathbb{F}=\{[\mathscr{G}, A] \times[\mathscr{H}, B]:[\mathscr{G}, A] \in \mathscr{T},[\mathscr{H}, B] \in \mathscr{U}\}$.

Then $\exists$ a subfamily $\mathbb{F}^{\prime}=\left\{\left[\mathscr{G}_{k}, A\right] \times\left[\mathscr{H}_{k}, B\right]: k \in \Delta\right\}$ of $\mathbb{F}$ such that $[\mathscr{F}, A \times B]=\bigsqcup_{k \in \Delta}\left[\left[\mathscr{G}_{k}, A\right] \times\left[\mathscr{H}_{k}, B\right]\right]$. Thus,

$$
\begin{aligned}
{\left[f_{\varphi_{\psi}}\right]^{-1}[[\mathscr{F}, A \times B]] } & =f_{\varphi_{\psi}}^{-1}\left[\sqcup_{k \in \Delta}\left[\left[\mathscr{G}_{k}, A\right] \times\left[\mathscr{H}_{k}, B\right]\right]\right] \\
& =f_{\varphi_{\psi}}^{-1}\left[\sqcup_{k \in \Delta}\left[\left(\Pi_{\varphi_{\psi^{A}}^{A}}^{X}\right)^{-1}\left[\mathscr{G}_{k}, A\right] \sqcap\left(\pi_{\varphi_{\psi^{B}}^{B}}^{Y}\right)^{-1}\left[\mathscr{H}_{k}, B\right]\right]\right] \\
& =\sqcup_{k \in \Delta}\left[\left(\pi_{\varphi_{\psi^{A}}^{X}}^{X} \circ f_{\varphi_{\psi}}\right)^{-1}\left(\left[\mathscr{G}_{k}, A\right]\right) \sqcap\left(\Pi_{\varphi_{\psi^{B}}^{B}}^{Y} \circ f_{\varphi_{\psi}}\right)^{-1}\left(\left[\mathscr{H}_{k}, B\right]\right)\right] \in \mathscr{W} .
\end{aligned}
$$

Therefore $f_{\varphi_{\Psi}}:[Z, C, \mathscr{W}] \rightarrow[X \times Y, A \times B, \mathscr{T} \times \mathscr{U}]$ is type-2 soft continuous.

Proposition 21. Let $[X \times Y, A \times B, \mathscr{T} \times \mathscr{U}]$ be the product space of two type-2 soft topological spaces $[X, A, \mathscr{T}]$ and $[Y, B, \mathscr{U}]$, where $\mathscr{U}$ contains all those type-2 soft sets of the form $\left[\widetilde{\left(\widetilde{Y_{0}}, B\right)}, B\right]$ where $Y_{0}$ be any subset of $Y$. Then for fixed $x_{0} \in X, \alpha_{0}, \alpha_{1} \in A$, the type-2 soft mapping $f_{\varphi_{\psi}}:[Y, B, \mathscr{U}] \rightarrow[X \times Y, A \times B, \mathscr{T} \times \mathscr{U}]$, defined by $f(y)=\left(x_{0}, y\right), \varphi(\beta)=$ $\left(\alpha_{0}, \beta\right)$ and $\psi(\beta)=\left(\alpha_{1}, \beta\right), \forall y \in Y, \forall \beta \in B$ is type-2 soft continuous.

Proof. Let $\Pi_{\varphi_{\psi^{A}}^{A}}^{X}:[X \times Y, A \times B, \mathscr{T} \times \mathscr{U}] \rightarrow[X, A, \mathscr{T}]$ and $\pi_{\varphi_{\psi^{B}}^{B}}^{B}:[X \times Y, A \times B, \mathscr{T} \times \mathscr{U}] \rightarrow[Y, B, \mathscr{U}]$ be the soft projection mappings. Then $\pi_{\varphi_{\psi^{A}}^{A}}^{A} \circ f_{\varphi_{\psi}}:[Y, B, \mathscr{U}] \rightarrow[X, A, \mathscr{T}]$ is a type-2 soft mapping such that $\left[\pi^{A} \circ f\right](y)=x_{0}, \forall y \in Y,\left[\varphi^{A} \circ \varphi\right](\beta)=\alpha_{0}$ and $\left[\psi^{A} \circ \psi\right]=\alpha_{1}, \forall \beta \in B$.

So, $\pi_{\varphi_{\psi^{A}}^{A}}^{A} \circ f_{\varphi_{\psi}}$ is a constant type-2 soft mapping and $\mathscr{U}$ contains all those type-2 soft sets of the form $\left[\widetilde{\left[\widetilde{Y}_{0}, B\right)}, B\right], Y_{0}$ be any subset of $Y$. Hence by Proposition 16, the soft mapping $\pi_{\varphi_{\psi^{A}}^{A}}^{A} \circ f_{\varphi_{\psi}}$ is type-2 soft continuous.

Again $\pi_{\varphi_{\psi^{B}}^{B}}^{B} \circ f_{\varphi_{\psi}}:[Y, B, \mathscr{U}] \rightarrow[Y, B, \mathscr{U}]$ is a type-2 soft mapping such that $\left[\pi^{B} \circ f\right](y)=y, \forall y \in Y,\left[\varphi^{B} \circ \varphi\right](\beta)=\beta$ and $\left[\psi^{B} \circ \psi\right]=\beta, \forall \beta \in B$. So, $\pi_{\varphi_{\psi^{B}}^{B}}^{B} \circ f_{\varphi_{\psi}}$ is an identity type-2 soft mapping. Hence by Proposition 15 , the soft mapping $\pi_{\varphi_{\psi^{B}}^{B}}^{B} \circ f_{\varphi_{\psi}}$ is type-2 soft continuous.

Therefore, by Proposition 20, for fixed $x_{0} \in X, \quad \alpha_{0}, \quad \alpha_{1} \in A$, the type-2 soft mapping $f_{\varphi_{\psi}}:[Y, B, \mathscr{U}] \rightarrow[X \times Y, A \times B, \mathscr{T} \times \mathscr{U}], \quad$ defined by $f(y)=\left(x_{0}, y\right), \quad \varphi(\beta)=\left(\alpha_{0}, \beta\right)$ and $\psi(\beta)=\left(\alpha_{1}, \beta\right), \forall y \in Y, \forall \beta \in B$ is type-2 soft continuous.

Proposition 22. Let $[X \times Y, A \times B, \mathscr{T} \times \mathscr{U}]$ be the product space of two type-2 soft topological spaces $[X, A, \mathscr{T}]$ and $[Y, B, \mathscr{U}]$, where $\mathscr{T}$ contains all those type-2 soft sets of the form $\left[\widetilde{\left(\widetilde{X_{0}}, A\right)}, A\right]$ where $X_{0}$ be any subset of $X$. Then for fixed $y_{0} \in Y, \beta_{0}, \beta_{1} \in B$, the type-2 soft mapping $f_{\varphi_{\psi}}:[X, A, \mathscr{T}] \rightarrow[X \times Y, A \times B, \mathscr{T} \times \mathscr{U}]$, defined by $f(x)=\left(x, y_{0}\right), \varphi(\alpha)=$ $\left(\alpha, \beta_{0}\right)$ and $\psi(\alpha)=\left(\alpha, \beta_{1}\right), \forall x \in X, \forall \alpha \in A$ is type-2 soft continuous.

Proof. Proof is similar to that of Proposition 21. 
Proposition 23. Let $f_{\varphi_{\psi}}:[X, A, \mathscr{T}] \rightarrow[X, A, T]$ and $g_{\xi_{\eta}}:[Y, B, \mathscr{U}] \rightarrow[Y, B, \mathscr{U}]$ be two type-2 soft continuous mappings. Then the type-2 soft mapping $h_{\rho_{\zeta}}:[X \times Y, A \times B, \mathscr{T} \times \mathscr{U}] \rightarrow[X \times Y, A \times B, \mathscr{T} \times \mathscr{U}]$ where $h(x, y)=(f(x), g(y)), \rho(\alpha, \beta)=(\varphi(\alpha), \xi(\beta))$ and $\zeta(\alpha, \beta)=(\psi(\alpha), \eta(\beta))$ is type-2 soft continuous.

Proof. Let $[\mathscr{F}, A \times B] \in(\mathscr{T} \times \mathscr{U})$. Then there exist $\left[\mathscr{G}_{i}, A\right] \in \mathscr{T}$ and $[\mathscr{H}, B] \in \mathscr{U}, i \in \Delta$ such that $[\mathscr{F}, A \times B]=\bigsqcup_{i \in \Delta}\left[\left[\mathscr{G}_{i}, A\right] \times\left[\mathscr{H}_{i}, B\right]\right]$. Since $f_{\varphi_{\psi}}$ and $g_{\xi_{\eta}}$ are type-2 soft continuous, it follows that $f_{\varphi_{\psi}}^{-1}\left[\left[\mathscr{G}_{i}, A\right]\right] \in \mathscr{T}$ and $g_{\xi_{\eta}}^{-1}\left[\left[\mathscr{H}_{i}, B\right]\right] \in \mathscr{U}$. Now $\forall(\alpha, \beta) \in(A \times B)$, we have

$$
\begin{aligned}
{\left[h_{\rho}^{-1}\left[\left(G_{i}, A\right) \widetilde{\times}\left(H_{i}, B\right)\right]\right](\alpha, \beta) } & =h^{-1}\left[\left[G_{i} \widetilde{\times} H_{i}\right](\rho(\alpha, \beta))\right] \\
& =h^{-1}\left[\left[G_{i} \widetilde{\times} H_{i}\right](\varphi(\alpha), \xi(\beta))\right] \\
& =h^{-1}\left[G_{i}(\varphi(\alpha)) \times H_{i}(\xi(\beta))\right] \\
& =f^{-1}\left[G_{i}(\varphi(\alpha))\right] \times g^{-1}\left[H_{i}(\xi(\beta))\right] \\
& =\left[f_{\varphi}^{-1}\left(G_{i}\right) \times g_{\xi}^{-1}\left(H_{i}\right)\right](\alpha, \beta) .
\end{aligned}
$$

Therefore, $h_{\rho}^{-1}\left[\left(G_{i}, A\right) \widetilde{\times}\left(H_{i}, B\right)\right]=\left(f_{\varphi}^{-1}\left(G_{i}\right), A\right) \widetilde{\times}\left(g_{\xi}^{-1}\left(H_{i}\right), B\right)$. Thus

$$
\begin{aligned}
{\left[h_{\rho_{\zeta}}^{-1}\left[\left[\mathscr{G}_{i}, A\right] \times\left[\mathscr{H}_{i}, B\right]\right]\right](\alpha, \beta) } & =h_{\rho}^{-1}\left[\left[\mathscr{G}_{i} \times \mathscr{H}_{i}\right](\zeta(\alpha, \beta))\right] \\
& =h_{\rho}^{-1}\left[\left[\mathscr{G}_{i} \times \mathscr{H}_{i}\right](\psi(\alpha), \eta(\beta))\right] \\
& =h_{\rho}^{-1}\left[\mathscr{G}_{i}(\psi(\alpha)) \widetilde{\times} \mathscr{H}_{i}(\eta(\beta))\right] \\
& =f_{\varphi}^{-1}\left[\mathscr{G}_{i}(\psi(\alpha))\right] \widetilde{\times} g_{\xi}^{-1}\left[\mathscr{H}_{i}(\eta(\beta))\right] \\
& =\left[f_{\varphi_{\psi}}^{-1}\left[\left[\mathscr{G}_{i}, A\right]\right](\alpha)\right] \widetilde{\times}\left[g_{\xi_{\eta}}^{-1}\left[\left[\mathscr{H}_{i}, B\right]\right](\beta)\right] \\
& =\left[f_{\varphi_{\psi}}^{-1}\left[\left[\mathscr{G}_{i}, A\right]\right] \times g_{\xi_{\eta}}^{-1}\left[\left[\mathscr{H}_{i}, B\right]\right]\right](\alpha, \beta) .
\end{aligned}
$$

Therefore,

$$
h_{\rho_{\zeta}}^{-1}\left[\left[\mathscr{G}_{i}, A\right] \times\left[\mathscr{H}_{i}, B\right]\right]=f_{\varphi_{\psi}}^{-1}\left[\left[\mathscr{G}_{i}, A\right]\right] \times g_{\xi_{\eta}}^{-1}\left[\left[\mathscr{H}_{i}, B\right]\right] \in(\mathscr{T} \times \mathscr{U})
$$

and hence

$$
h_{\rho_{\zeta}}^{-1}[[\mathscr{F}, A \times B]]=h_{\rho_{\zeta}}^{-1}\left[\bigsqcup_{i \in \Delta}\left[\left[\mathscr{G}_{i}, A\right] \times\left[\mathscr{H}_{i}, B\right]\right]\right]=\bigsqcup_{i \in \Delta}\left[\left[\mathscr{G}_{i}, A\right] \times\left[\mathscr{H}_{i}, B\right]\right] \in(\mathscr{T} \times \mathscr{U})
$$

Therefore, the type-2 soft mapping $h_{\rho_{\zeta}}:[X \times Y, A \times B, \mathscr{T} \times \mathscr{U}] \rightarrow[X \times Y, A \times B, \mathscr{T} \times \mathscr{U}]$, where $h(x, y)=(f(x), g(y)), \rho(\alpha, \beta)=(\varphi(\alpha), \xi(\beta))$ and $\zeta(\alpha, \beta)=(\psi(\alpha), \eta(\beta))$ is type-2 soft continuous.

\section{Conclusion and future work}

In this paper, concepts of type-2 soft topology is introduced and studied some of its important properties. There is an wide scope for further research to extend it in topological group theory which have many applications in abstract integration theory viz. Haar measure, Haar integral etc. and also in manifold theory through the development of Lie groups.

\section{Acknowledgement}

The author express his sincere thanks to the reviewers for their valuable and constructive suggestions which have improve the presentation of the paper to a great extent. The author is also thankful to the Editor-in-Chief and the Associate Editors for their valuable advice. 


\section{Competing interests}

The authors declare that they have no competing interests.

\section{Authors' contributions}

All authors have contributed to all parts of the article. All authors read and approved the final manuscript.

\section{References}

[1] Aktas, H. and Cagman, N., Soft sets and soft groups, Inform. Sci., 177 (2007) 2726-2735.

[2] Bhabitha, K. V. and Sunil, J. J., Soft relations and functions, Comput. Math. Appl., 60 (2010) 1840-1849.

[3] Cagman, N., Karatas, S. and Enginoglu, S., Soft topology, Comput. Math. Appl., 62 (2011) 351-358.

[4] Chatterjee, R., Majumda, P. and Samanta, S. K., Type-2 soft sets, J. Intell. \& Fuzzy Systems, 29 (2015) 885-898.

[5] Hazra, H., Majumdar, P. and Samanta, S. K., Soft topology, Fuzzy Inform. Eng., 4(1) (2012) 105-115.

[6] Hussain, S. and Ahmad, B., Some properties of soft topological spaces, Comput. Math. Appl. 62 (2011) 4058-4067.

[7] Kharal, A. and Ahmad, B., Mappings on soft classes, New Math. Nat. Comput., 7(3) 471-481.

[8] Maji, P. K. and Roy, A. R., An application of soft sets in a decision making problem, Comput. Math. Appl., 44 (2002) 1077-1083.

[9] Majumdar, P. and Samanta, S. K., On soft mappings, Comput. Math. Appl. 60 (2010) 2666-2672.

[10] Majumdar, P. and Samanta, S. K., Similarity measure of soft sets, New Math. Nat. Comput., 4(1) (2008), 1-12.

[11] Molodtsov, D., Soft Set Theory-First Results, Comput. Math. Appl., 37 (1999) 19-31.

[12] Nazmul, Sk. and Samanta, S. K., Neighbourhood properties of soft topological spaces, Annl. Fuzzy Math. Inform., 6(1) (2013) $1-15$.

[13] Nazmul, Sk. and Samanta, S. K., Soft topological soft groups, Mathematical Sciences, 6:66 (2012) 1-10.

[14] Nazmul, Sk. and Samanta, S. K., Generalized group soft topology, Annl. Fuzzy Math. Inform., 9(5) (2015) 783-800.

[15] Nazmul, Sk. and Samanta, S. K., Some properties of soft groups and fuzzy soft groups under soft mappings, accepted at PJM, on December, 2016.

[16] Nazmul, Sk., Type-2 soft groups, Communicated at Mathematical Sciences, Springer on September, 2016.

[17] Shabir, M. and Naz, M., On soft toplogical spaces, Comput. Math. Appl., 61(7) (2011) 1786-1799.

[18] Zadeh, L. A., Fuzzy sets, Inform. And Control, 8 (1965), 338-353.

[19] Zadeh, L. A., The concept of linguistic variable and its application to approximate reasoning-I, Inform. Sci.(1975), 199-245. 